\title{
Calidad de servicio y recurso humano: caso estudio tienda por departamentos
}

\author{
Hernández de Velazco, Judith* \\ Chumaceiro, Ana Cecilia** \\ Atencio Cárdenas, Edith***
}

\section{Resumen}

Calidad de Servicio es una estrategia básica para enmarcar la estructura y funcionalidad organizativa dentro de parámetros que consideren las expectativas y necesidades clientelares, la importancia del recurso humano para dar respuesta a estas exigencias debe ser determinante para garantizar la calidad de los servicios que ofertan. Por lo tanto, el propósito del artículo es revisar resultados de una investigación preliminar que tuvo como objetivo evaluar la calidad de servicio y la importancia del recurso humano específicamente los empleados de atención al cliente en una tienda por departamentos del Estado Zulia. La investigación fue descriptiva y la muestra fue probabilística-intencionalcasual escogida entre clientes de dos tiendas del grupo ubicadas en la ciudad de Maracaibo, el instrumento aplicado fue un cuestionario de elección múltiple. Se evidencia que la atención al cliente como servicio es percibida por los clientes como regular-mala por lo tanto debe ser mejorada en sus áreas críticas, también se determinaron factores de interés según preferencias clientelares todos relacionados con el recurso humano, se recomienda diseñar un programa de entrenamiento en Calidad de Servicio para los empleados.

Palabras clave: Calidad de servicio, recurso humano, atención al cliente, evaluación del servicio.

\section{Recibido: 20-06-08. Aceptado: 01-07-09}

* Lic. Ciencias Políticas y Administrativas. Master en Administración de Empresas. Candidata a Doctora de Ciencias Sociales. Mención Gerencia. PPI. Docente-Investigadora UNERMB. Coordinadora General del Centro de Estudios e Investigaciones Socioeconómicas y Políticas (CEISEP). Correo: lasanas23@cantv.net

** Lic. Administración. Mención Gerencia Industrial. Participante en el Postgrado Gerencia Tributaria. Docente-Investigadora Novel. Correo: ceciliahernanz@hotmail.com

*** Lic. Administración; Magíster en Gerencia de Empresas, mención: Servicios Administrativos. Candidata a Doctora en Administración de Empresas. PPI. Coordinadora Administrativa de Revista Venezolana de Gerencia delCentro de Estudios de la Empresa de FACES Universidad del Zulia. E-mail: edithsoraida@yahoo.es 


\title{
Quality of Service and Human Resources: Case Study of a Department Store
}

\begin{abstract}
Quality of service is a basic strategy to place structure and organizational functionality within parameters that consider the customers' expectations and needs; the importance of human resources to respond to these demands ought to be determinant to guarantee the quality of services offered. The intention of this article is to review results of a preliminary investigation whose objective was to evaluate the quality of service and the importance of human resources, specifically the employees who attend clients in a department store in the State of Zulia. The research was descriptive and the sample was probabilistic-intentional-casual, chosen from clients of two stores in the group located in the city of Maracaibo. The applied instrument was a multiple choice questionnaire. It demonstrated that the attention to the client service is perceived by clients as moderate to bad; therefore it must be improved in critical areas. Also, interest factors were determined according to customer preferences, all related to human resources. The study recommends designing a quality of service training program for the employees.
\end{abstract}

Key words: Quality of service, human resource, attention to the client, service evaluation.

\section{Introducción}

La gerencia contemporánea define conceptos que permiten a las organizaciones adecuarse a las realidades del momento, herramientas para acceder exitosamente a un mercado altamente competitivo, donde la ventaja puede ser definida por diversos criterios de apreciación de los consumidores y por la evaluación que hacen sobre el servicio recibido. Entendiendo, que cualquier organización ofrece algún tipo de servicio relacionado con la atención al cliente.

Frente a esa dinámica, surgen planteamientos vinculados a la Calidad del Servicio como concepto transformativo, filosofía, valores y actitudes que deben predominar en un negocio. Por lo tanto, asumimos Calidad de Servicio como instrumento competitivo, cultura organizativa, proceso de mejora continuo, compromiso global (empleados, gerencia y accionistas con los clientes), ventaja competitiva y estrategia empresarial.

Pero, para alcanzar estos criterios, se requiere estructurar un plan de acción y desarrollo del recurso humano involucrado en la atención al cliente, quien se constituye en el elemento principal para afianzar la cultura, el compromiso, y la estrategia de la calidad del servicio. El recurso humano, que interactúa directamente con los clientes representa, transmite la calidad del negocio en la generalidad de todos sus procesos, su interrelación lo constituye en el eslabón intermedio entre empresa y consumidores, por lo tanto, inciden sobre las preferencias de consumo.

Considerando lo anterior, es pertinente plantear en este discurso, los resultados de una investigación preliminar (año 2007) que realizamos relacionada con el tema, donde se evaluó la calidad del servicio ofrecida por los empleados 
de ventas de una tienda por departamentos del Estado Zulia a través de la aplicación de una encuesta; se evidencia, como plantearemos, factores críticos de calidad de servicio según sus clientes y que deben ser mejorados, además se definieron expectativas clientelares que deben ser consideradas en el corto y mediano plazo. Como propósito demostraremos la importancia del Recurso Humano para la Calidad de Servicio.

\section{Aproximaciones teóricas de la calidad del servicio}

Hablar de Calidad de Servicio como concepto la define como la capacidad de satisfacer al cliente en sus necesidades, expectativas y requerimientos (Juran 1995), es la satisfacción que se le proporciona al cliente a través del servicio.

Las empresas $u$ organizaciones empresariales en cualquier momento de su proceso de venta, sea de bienes, productos o servicios (que es el caso de la investigación) realizan contactos directos con los compradores o clientes, a través de personas o empleados que facilitan, intermedian, ejecutan, interpretan sus demandas para ser satisfechas.

En efecto, el servicio con calidad ha sido y es un imperativo para todos, porque la preferencia de los clientes por una determinada empresa le facilita posicionarse del mercado de su interés y le otorga ventaja competitiva frente a otros.

En pleno siglo XXI, el mercado con diversidad de opciones, oportunidades $y$ alternativas para los consumidores obliga a las empresas además de invertir en tecnología de punta, en recurso humano de amplio perfil, en redes de conocimiento y aprendizaje, en investigación e innovación, todas las cuales redundan en un mejor desempeño organizacional pero que deben confluir en la calidad del servicio al cliente, como momento final. Esta tendencia es así en la actualidad como también lo ha sido desde los inicios del desarrollo histórico del concepto de calidad.

\subsection{Antecedentes de la calidad del servicio}

Entre la segunda mitad del S. XVIII y principios del S. XIX se produce la revolución industrial con todas sus implicaciones tecnológicas, sociales, económicas y laborales, período histórico a partir del cual, se concibe la calidad como elemento de medición sobre la producción.

Calidad se infiere según dos principios: el de inspección del producto y el de reconocimiento de las habilidades artesanales; en la inspección, el consumidor se orientaba por sus propios conocimientos, experiencias o intuición; en el reconocimiento del artesano, el cliente confiaba en las habilidades, reputación y prestigio del productor.

La calidad evoluciona según Bounds (1994), a través de cuatro etapas en el siglo XIX; 1) la inspección; 2) la etapa del control estadístico del proceso (década del treinta), 3) aseguramiento de calidad (década de los cincuenta); y 4) administración o gestión de la calidad total (TQM, década del ochenta), con esta última, se considera las necesidades del consumidor, reconociendo el efecto estratégico de la calidad como una oportunidad competitiva.

Es determinante que varios eventos sociales influyen sobre la aplicación 
de Calidad, entre los cuales se cuentan: el reconocimiento estatutario de la propiedad privada, el establecimiento de límites económicos para los ciudadanos, el sistema de factorías, la creación de gremios artesanales y para 1900 la aparición de gremios profesionales con el taylorismo y a la postre con el fordismo. De tal manera que según Juran y Grina (1999), fueron surgiendo herramientas que tuvieron utilidad en el área productiva, como; especificaciones por muestras, mediciones e instrumentos de medidas, estandarización.

Adicionalmente, el crecimiento de los aparatos productivos y la complejidad de las redes de servicios evidenciaron la necesidad de establecer altos rendimientos de calidad. La complejidad de los servicios en los sectores que suministran energía, comunicaciones, transporte y comercialización (sector objeto de la investigación), fueron presionando a la aplicación extensiva de la calidad.

Después de la segunda guerra mundial, los japoneses iniciaron un giro estratégico dirigido a la cultura laboral desde el empresariado y con mayor énfasis en los empleados para desarrollar un nuevo concepto de prestación de servicio.

Para Pestana y García (2003:1314), Calidad total..."Hoy por hoy, se ha convertido en un elemento clave necesario e indispensable para el éxito empresarial. Sus representantes reconocidos a nivel mundial (W. Edwards Deming, Kaoura Ishikawa; Joseph Juran, Phillip Crosby y Conway) coinciden en que la calidad gerencial, es precisamente una vía para el mejoramiento...y la posición competitiva en el mercado."
Por otra parte, en la actualidad los resultados sobre participación del factor humano en la Calidad son concluyentes. Según Atencio y González (2006), la calidad del servicio es responsabilidad de toda la organización donde se involucre a todo el que se relaciona directa e indirectamente con los clientes y el servicio que se le presta, porque es lo que en definitiva valora el cliente como una totalidad y no como una propiedad específica del producto, bien o servicio que le ofrecen.

Deming (1989), señala a la calidad como la producción eficiente que el mercado está demandando, siendo la base de esto la productividad y la competitividad; lo que se puede resumir en la búsqueda del mejoramiento continuo. Por otra parte, para Juran (1995), la calidad es la adecuación al uso de lo que se esta ofreciendo, agregando además satisfacción del cliente; considerando al cliente como todo aquel a quien un producto o proceso impacta.

Podemos decir que la Calidad de Servicio es entonces; un instrumento competitivo que requiere una cultura organizativa cónsona, un compromiso de todos, dentro de un proceso continuo de evaluación y mejoramiento, para ganar la lealtad del cliente y diferenciarse de la competencia como estrategia de beneficio.

Juran y Gryna (1999), plantean que la calidad de un producto o servicio, es la caracterización del artículo o servicio obtenido en el proceso de producción o servicio que determina el grado de su correspondencia con el conjunto de exigencias establecidas por los consumidores. 


\section{Enfoque de calidad de servicio}

Los orígenes del modelo gerencial basado en Calidad del servicio tiene su mayor representante en Jan Carlzon, (1981) revolucionó con su diseño, desarrollo y prestación del servicio excelente, cuyo supuesto inicial era hacer extrovertida a la organización, para controlar la experiencia del cliente y definir los momentos de verdad en la prestación del servicio.

Del modelo gerencial surgen los principios de:

- Controlar las experiencias del cliente en todos los puntos durante el ciclo del servicio

- Organización orientada hacía el cliente

- Sistemas amables para el cliente

- Cultura y ambiente de trabajo que refuerce la idea de que el cliente esta primero.

Por su parte las organizaciones que aplican gerencia de servicio, trabajan con un estilo de dirección comprometido profundamente con la filosofía de calidad, acorde con la necesidad de trabajar para el cliente. Este estilo de dirección busca, entre otras cosas: dotar a los trabajadores de las herramientas necesarias que les permitan hacer un excelente trabajo; lograr un personal capacitado y comprometido con la calidad; aplicar la objetividad en la evaluación de los empleados y de la organización por parte de los clientes.

La Calidad expone siete (7) imperativos sobre todo para el desarrollo del servicio, que según Whiteley (1992), son:
1. Necesidad de una visión orientada a la conservación del cliente, además de objetivos claros y compartidos.

2. Omnipresencia del cliente en la organización

3. Recopilar información, herramientas, aplicaciones sobre Calidad del Servicio

4. Confiar en las competencias de los empleados para ofrecer un buen servicio al cliente

5. Mejorar los procedimientos entre los clientes y la empresa

6. Medir el desempeño organizacional con relación a la Calidad

7. Actuar y vivir para el cliente

De estos imperativos planteados se hace necesario un cambio cultural en los patrones de venta porque no solo es requerida la calidad del producto sino que ahora es necesaria también la calidad del servicio que se presta. En conclusión las empresas tienen que complacer y satisfacer las necesidades globales de los clientes, para lo cual necesita un recurso humano competente.

En términos generales, en la prestación de un servicio lo que hace y marca la diferencia entre varias alternativas de competidores, o entre una oferta y otra es el nivel de servicio, tanto alrededor del producto como por la riqueza de los componentes constitutivos del servicio, bien sea que éste último tenga relación con la atención personalizada al cliente, la bienvenida, el proceso mismo de la venta, ó la post venta.

Para Calidad de Servicio se maneja cinco (5) dimensiones que están determinadas en su totalidad por el Recurso Humano, como son: la tangibilidad en los aspectos visibles del Servicio, la atención 
al cliente por parte de los empleados; confiabilidad, en la empresa y en su gente; responsabilidad de la gerencia y de los empleados sobre lo qué oferta y cómo se oferta; seguridad como garantía de compra y respuesta frente a las especificaciones; y empatía con el mercado, por el deseo de comprender las necesidades, requerimientos y expectativas clientelares (Whiteley;1992)

Tal como lo expresan Kaplan y Norton (2000:6) "El proceso interno se refiere a los procesos críticos que permitan acercar propuestas para retener a los clientes y satisfacer expectativas de rendimientos financieros. Por ejemplo, en lugar de centrarse en la entrega de pedidos, se comienza a crear valor desde el pedido del cliente, luego la recepción y finalmente la entrega.

La última perspectiva es la de aprendizaje y crecimiento. Proviene de tres fuentes: las personas, los sistemas y los procedimientos. Al igual que los clientes, a los empleados también es preciso retenerlos, satisfacerlos, entrenarlos y fortalecer sus habilidades. Quizás éste sea el punto más difícil de entender en Argentina.

\section{El factor humano}

El servicio en sí implica o involucra personas que tratan con personas. Entre todos los componentes que intervienen en las actividades del servicio, el factor humano parece ser el más crítico. No obstante la amplia variedad de servicios con la que entramos en contacto, el denominador común es el factor humano. La percepción que nos queda del servicio, está íntimamente ligada a las personas que lo suministraron.

Lo que el cliente recibe y el grado en que se satisfagan sus necesidades y expectativas, dependen del servicio. Según Rodriguez (2000), en Norma ISO 9000 versión 2000 , se define la satisfacción del cliente, como: percepción que tiene el cliente sobre el grado en que se han cumplido sus requisitos; definiendo a su vez requisito como: necesidad o expectativa establecida, generalmente implícita u obligatoria.

De todo esto mencionado, se conjugan las condiciones que deciden la trascendencia del servicio y la permanencia del cliente. Pero hay que considerar muy atentamente que los clientes les gustan los dispositivos que traigan comodidad y ahorro de tiempo. Como también desean un ser humano que este cerca cuando el sistema en general no hace lo que se espera.

Los Recursos Humanos en definitiva, son los responsables finales de satisfacer al cliente, son los que tienen en sus manos la posibilidad de lograr que su empresa aprenda a vivir para el servicio (Peters y Waterman, 1987).

En realidad el empleado es el eslabón fundamental para anclar el cliente en la preferencia de la empresa, para asistirlo en sus necesidades y para ser el decodificador de sus mensajes, de forma tal que se pueda satisfacer sus expectativas plenamente.

El Factor Humano de las organizaciones, determina la percepción de los clientes, condiciona el momento de verdad, señalado este como el preciso instante en que el cliente hace contacto con 
la empresa y dependiendo de su experiencia se forma una opinión sobre Calidad del Servicio.

Braidot y otros (2003), identifican características o elementos comunes que plantean los especialistas del área para una mejor calidad del servicio, entre los cuales resaltan:

- La orientación al cliente, como la satisfacción de sus necesidades y expectativas. Considerado como un concepto estratégico, que exige una constante sensibilidad y atención a la información del mercado, y una gran capacidad de adaptación interna para poder ofrecer una respuesta ágil y rápida a las demandas detectadas.

- La toma de decisiones basadas en el análisis de hechos y datos. Esto exige el despliegue de los indicadores a lo largo de la organización para suministrar la información necesaria para asumir las transformaciones necesarias orientas al cliente.

- La gestión de los recursos humanos, considerados como el recurso clave de la empresa, siendo crucial el pleno desarrollo de sus capacidades y su participación e integración en el proyecto de empresa, el trabajo en equipo y la utilización de herramientas y técnicas adecuadas a cada nivel de la organización.

La relación que se establece entre el cliente y el empleado define el contexto y el tipo de relación que caracterizará al Servicio, por ello es importante lograr una alta congruencia en esta interrelación. Congruencia que estará determinada por la similitud de valores y preferencias que exista entre los involucrados.
Cuando los empleados se capacitan para convertirse en investigadores inmediatos del mercado, la organización obtiene una visión muy cercana del cliente, además que la mejora tiene un doble efecto, en cuanto al conocimiento del mercado y en cuanto a la motivación de los empleados.

En concordancia, las variables relacionadas con Calidad del Servicio giran en torno a la eficacia en el logro del objetivo de satisfacción al cliente; efectividad en el cumplimiento del compromiso organizacional; eficiencia en el uso de recursos que se utilicen para prestar el Servicio; y el desarrollo, evaluación y mejoramiento del recurso humano. Los indicadores de desarrollo, evaluación y mejoramiento del recurso humano incluyen por su parte clima organizacional pertinente al servicio, valoración del servicio, programas de entrenamiento, entre otros.

Considerando todo lo anterior y a efectos de analizar los resultados de la evaluación del caso estudio tienda por departamento; se considera a la variable de desarrollo, evaluación y mejoramiento del recurso humano como factor determinante de forma directa en la satisfacción o insatisfacción del los clientes; y al indicador de valoración (evaluación) de servicio por parte de los clientes como determinante para programar el entrenamiento del recurso humano en atención al público, para mejorar la calidad del servicio.

\section{Caso de estudio. Empresa del sector comercial}

La evaluación Servicio se realizó a una empresa dedicada a la comercialización de mercancía y productos (juguetes, 
textiles, artículos de hogar, entre otros) relacionada con los diferentes departamentos que le componen. Su ubicación geográfica se extiende por el Estado Zulia, y en la ciudad de Maracaibo se ubican dos tiendas, las cuales representan la mayor inversión en infraestructura, metros cuadrados, mercancía, y en personal, pero al mismo tiempo representan los mayores porcentajes de venta y retorno de inversión.

El objetivo de la investigación fue evaluar la Calidad de Servicio prestada por los empleados en el área de venta de los diferentes departamentos de esta tienda. Para indagar sobre Calidad de su servicio fue necesario extraer la mayor información posible sobre; atención al cliente, preferencias clientelares, y definición de importancia en los criterios de servicio prestados tales como; solución de problemas, información adecuada, rapidez en la venta, y otros.

La condición de evaluar la calidad del Servicio, dio como resultado el análisis y diagnóstico de los requerimientos de la clientela a la que se le presta el servicio, esto trae como consecuencia la necesidad de establecer como población a los clientes de las tiendas ( 5 en total) ubicadas en el Estado Zulia. Se definió una muestra probabilística-intencional-casual escogida de entre las personas que visitan las dos (2) tiendas de Maracaibo, seleccionadas estas tiendas por acumular las mayores transacciones y ventas del conjunto, para la tienda que identificaremos como 1 , se seleccionaron 150 clientes (tienda estrella en ventas y mayores transacciones y visitas de clientes), y para la tienda identificada como 2 se seleccionaron 100 clientes (segunda mejor tienda en ventas, transacciones y visitas de clientes).

El instrumento de recolección de datos, fue un cuestionario (Hernández y otros; 2006) de selección múltiple, donde se evaluó; atención al cliente, preferencias clientelares, rapidez en la venta, distribución de mercancías y productos(desde textil hasta juguetes), decoración y otros. Antes de aplicar el instrumento a la muestra seleccionada se validó (validez de contenido) a través de un juicio de expertos (grupo de profesionales con postgrado en el área.) quienes determinaban si los ítems que trataban de medir las variables y dimensiones eran exhaustivos, confiables y adecuados para realizar la investigación sobre la calidad de servicio. Por razones propias del estudio el instrumento se construyo en base a una escala de Likert, pero también se utilizaron preguntas abiertas, cerradas y de selección múltiple (Arias, 2006), dado que se buscaba información socioeconómica, y demográfica de los clientes, finalmente esas respuestas después fueron cruzadas con los resultado obtenidos con la escala de Likert. El instrumento se aplico en el año 2007.

Los resultados de la encuesta aplicada permitieron; en primer lugar, la evaluación de los clientes, donde se obtuvo una apreciación de regular a mala en cuanto a la atención al cliente y el servicio, en general; $y$ en segundo término, obtener referencias vinculadas a las perspectivas y apreciaciones de los clientes, en concordancia con lo mencionado desde el inicio, determinar factores de interés clientelares para ser considerados en un programa de entrenamiento en calidad de servicio. 
Los resultados revisados a continuación no expresan la totalidad de todos los resultados obtenidos en la evaluación general realizado por los clientes a las dos tiendas, evidentemente se muestran los que guardan relación y coherencia con la estructura y propósito de la presente disertación

Al analizar los resultados se reflejan ciertas características de la calidad de servicio de las tiendas, recordando que la calidad de servicio es un conjunto de atributos que exigen los consumidores (Ju- ran y Grina; 1999), características y factores que fueron definidos rigurosamente en su momento incluyendo dentro de ellas (atención al cliente, variedad de productos y mercancías, facilidades en el proceso de pago, y otros); en consecuencia se evidencia:

En el Cuadro y Gráfico 1, como vemos a continuación, se reflejan la clasificación realizada por los clientes de los diferentes aspectos que caracterizan a la tienda 1, la mejor clasificación como excelente es para la variedad de productos

\section{Cuadro 1}

TIENDA 1. Clasificación de aspectos según clientes

\begin{tabular}{lcccc}
\hline & Excelente & Bueno & Regular & Malo \\
\hline Variedad de productos & $53(35 \%)$ & $91(61 \%)$ & $6(4 \%)$ & - \\
Calidad del Servicio & $14(9 \%)$ & $37(25 \%)$ & $99(66 \%)$ & - \\
Decoración & $46(31 \%)$ & $88(59 \%)$ & $15(10 \%)$ & - \\
Distribución de productos & $70(47 \%)$ & $74(49 \%)$ & $6(4 \%)$ & - \\
Precios & $24(16 \%)$ & $102(68 \%)$ & $24(16 \%)$ & - \\
Estacionamiento & $32(21 \%)$ & $80(53 \%)$ & $18(12 \%)$ & $4(3 \%)$ \\
\hline
\end{tabular}

Fuente: Elaboración propia (2007).

\section{Gráfico 1}

\section{Clasificación de aspectos según clientes}

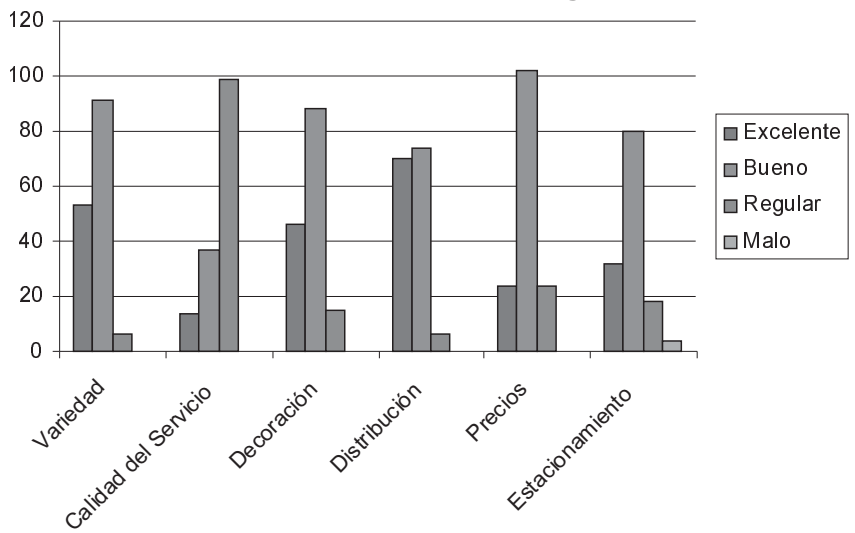

Fuente: Elaboración propia. 
y mercancías, lo que es consistente con una tienda por departamentos donde se consigue desde textiles hasta juguetería. En la clasificación regular la Calidad de Servicio obtuvo el mayor porcentaje $66 \%$, revelando insatisfacción clientelar en cuanto al servicio que le ofrecen, es decir que es una debilidad o área crítica dada la importancia del aspecto planteada en el discurso.

Los resultados para la tienda 2 según el Cuadro y Gráfico 2, tienden a reflejar percepciones similares de los clientes con relación a la Calidad de Servicio considerada como regular en un $70 \%$, como excelente a la Distribución de productos en un $47 \%$ y como bueno la variedad de productos y mercancías en los diferentes departamentos con $63 \%$.

En general, para ambas tiendas las mejores percepciones no son por brindar un mejor servicio, sino por valores agregados a la constitución propia del negocio, como las diversidad y variedad de mercancías, a la distribución de los departamentos con sus productos, a los

\section{Cuadro 2}

TIENDA 2. Clasificación de aspectos según clientes

\begin{tabular}{lcccc}
\hline & Excelente & Bueno & Regular & Malo \\
\hline Variedad de productos & $35(35 \%)$ & $63(63 \%)$ & $2(2 \%)$ & - \\
Calidad del Servicio & $4(4 \%)$ & $26(26 \%)$ & $70(70 \%)$ & - \\
Decoración & $32(32 \%)$ & $56(56 \%)$ & $12(12 \%)$ & - \\
Distribución de productos & $47(47 \%)$ & $49(49 \%)$ & $4(4 \%)$ & - \\
Precios & $10(10 \%)$ & $77(77 \%)$ & $13(13 \%)$ & - \\
Estacionamiento & $37(37 \%)$ & $35(35 \%)$ & $8(8 \%)$ & $18(18 \%)$ \\
\hline
\end{tabular}

Fuente: Elaboración propia (2007).

\section{Gráfico 2}

\section{Clasificación de aspectos según clientes}

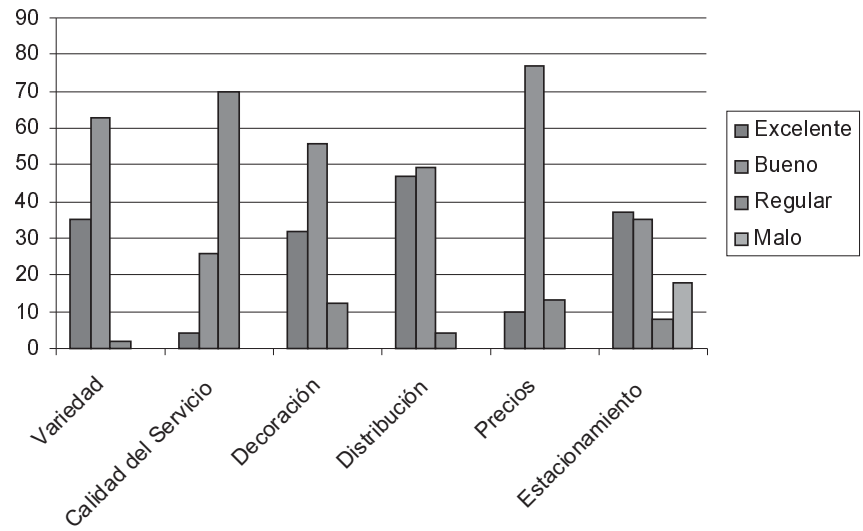

Fuente: Elaboración propia. 
precios considerados como buenos en casi un $78 \%$.

De estas tendencias se puede deducir que la diferencia entre los aspectos es de significativa importancia, porque aunque los clientes evidencien preferencias por precios, por ejemplo, evidentemente que la atención no es un factor de apoyo para la empresa. Lo que se demuestra en el Gráfico 3, con un resultado para la tienda 1 de regular $(40 \%)$ y de malo $(30 \%)$, el $70 \%$ de la muestra para la atención al cliente le pondero como regular-malo.

Igualmente, para la tienda 2 según el Gráfico 4, la evaluación para la atención al cliente es de regular-mala en un $70 \%$.

Los resultados que se presentan a continuación continúan consistentemente con la misma tendencia negativa para

\section{Gráfico 3}

TIENDA 1. En cuanto a atención al cliente ¿cómo evalúa al empleado?

\section{Excelente}

$15(10 \%)$

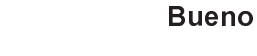

$30(20 \%)$

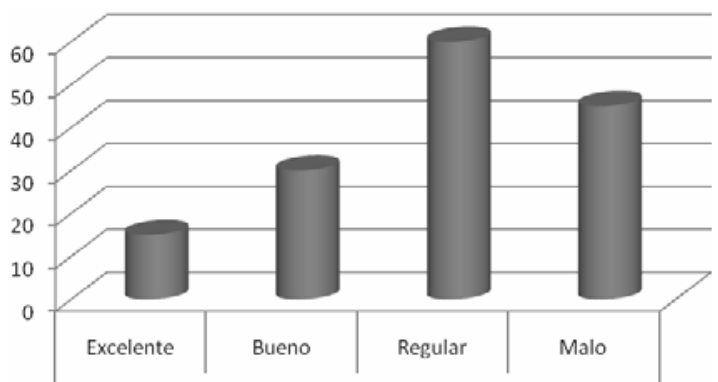

Fuente: Elaboración propia (2007).
Regular

Malo

$45(30 \%)$

\section{Gráfico 4}

TIENDA 2. En cuanto a atención al cliente ¿cómo evalúa al empleado?

\section{Excelente}

$11(11 \%)$
Bueno

$19(19 \%)$
Regular

$38(38 \%)$

Malo

$32(32 \%)$

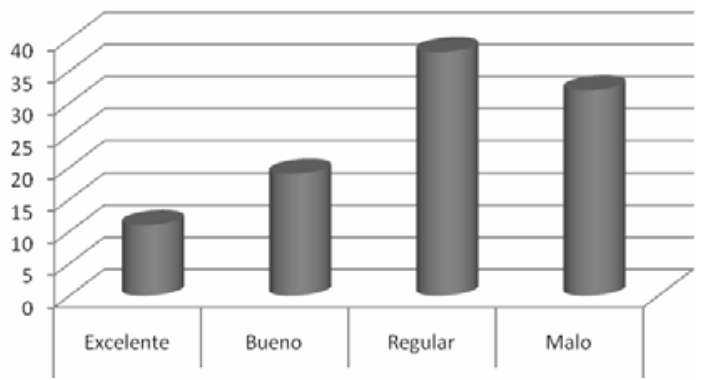

Fuente: Elaboración propia (2007). 
la percepción de los clientes en cuanto a la Calidad de Servicio, porque al sugerir cambiar la atención al cliente en un porcentaje del 60 , indica que no se siente satisfecho el cliente con la atención prestada.

Pero al mismo tiempo, el Cuadro 3 y el Gráfico 5 señalan los requerimientos de los clientes, información necesaria y oportuna para mejorar la Calidad del Servicio en esas áreas. Para la consecución del compromiso organizacional con la Calidad del Servicio, es pertinente conocer al cliente, sobre todo cuando se pretende trabajar con el fin de identificar las expectativas, demandas y exigencias del mercado actual y del futuro. Es importante por lo tanto considerar los factores claves que arroja esta evaluación tales como la atención al cliente, agilizar el proceso de venta (pago en las cajas registradoras, diversificar las formas de pago, mejorar el ambiente (clima, aires acondicionados), entre otros, para mejorar el servicio.
La valoración de estos factores define las preferencias clientelares que en el corto, mediano y largo plazo pueden favorecer o desfavorecer la ventaja competitiva, a continuación se presentan resultado relacionados con las expectativas clientelares.

En definitiva también puede apreciarse, que los aspectos relacionados con la intervención de empleados de forma directa con el cliente representan casi un $30 \%$ como cajeros y atención al cliente, los otros incluyen la participación de los empleados de forma indirecta, lo que deja demostrado la importancia del Recurso Humano para ofrecer Calidad de Servicio.

\section{Conclusiones}

En las tiendas evaluadas, la Calidad de Servicio referida a la atención al público es considerada de regular a mala, aunque no pueden considerarse inexistente la práctica del Servicio por parte de

\section{Cuadro 3}

(AMBAS TIENDAS) ¿Qué sugiere cambiar en las tiendas?

\begin{tabular}{lc}
\hline \multicolumn{1}{c}{ Cambios } & Frecuencia \\
\hline Ampliar numero de cajas registradoras y de cajeros & $19(7,6 \%)$ \\
Agilizar Cancelación de cheque y tarjeta crédito & $38(15,2 \%)$ \\
Atención al cliente & $60(24 \%)$ \\
Mejorar Variedad de Productos & $30(12 \%)$ \\
Ampliar Estacionamiento & $27(10,8 \%)$ \\
Mejorar Entradas de las Tiendas & $19(7,6 \%)$ \\
Mejorar capacidad de aires acondicionados & $15(6 \%)$ \\
Señalización de Departamentos. & $8(3,2 \%)$ \\
No sabe/No responde & $14(5,6 \%)$ \\
\hline
\end{tabular}

Fuente: Elaboración propia (2007). 


\section{Gráfico 5 \\ (AMBAS TIENDAS) ¿Qué sugiere cambiar en las tiendas?}

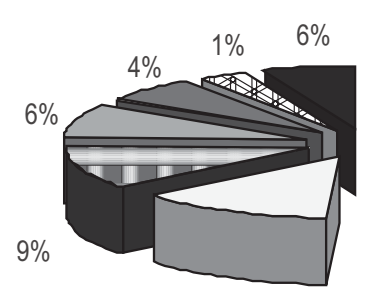

$14 \%$

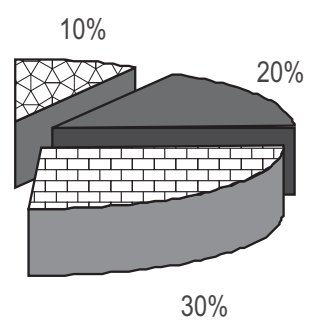

Ampliar número de cajas
registradoras
$\square$ Agilizar Cancelación de
cheque y tarjeta crédito
$\square$ Atención del Personal
$\square$ Mejorar Variedad de
Productos
$\square$ Ampliar Estacionamiento
$\square$ Mejorar Entradas de las
Tiendas
$\square$ Mejorar capacidad de
aires acondicionados
Señalización de
Departamentos.
No sabe/No responde

Fuente: Elaboración propia (2007).

los empleados de ventas, no es una fortaleza ni ventaja competitiva.

Por el contrario, los resultados reflejaron áreas críticas; una, en la atención al cliente señalando apatía, falta de cortesía, desconocimiento de la mercancía y precios; y otra, en el proceso de venta (retrasos en las cajas registradoras); generando ambas molestia e insatisfacción en los compradores.

En consecuencia y en general, el enfoque de Calidad del Servicio, debe ser asequible, manejable y aceptado por los empleados de cualquier empresa. Por lo tanto, la Calidad debe concentrarse inicialmente en la transformación de la organización como un todo, desde la gerencia, a los accionistas, proveedores y empleados.
También es necesario que los sistemas a implantar en las Empresas deben estructurarse congruentemente con los propósitos trazados en función del recurso humano requerido, contratado y utilizado para atender al público.

Diversos instrumentos permiten a las empresas mantenerse cerca de sus clientes, lo importante es planificar, programar y realizar seguimientos constantemente al mercado, para fortalecer y mejorar el Programa de Calidad existente.

Las herramientas para el desarrollo de un Recurso Humano competente para el Servicio, incluye; cultura organizacional para y por el cliente, formación y entrenamiento para potenciar y fortalecer habilidades y destrezas necesarias para ofrecer un servicio con calidad, entre otras. 
Para finalizar, es recomendable crear un Programa de Entrenamiento para el personal, que debe estar orientado en tres sentidos: acorde a los objetivos organizacionales y del recurso humano, en función de las necesidades determinadas por la evaluación clientelar, y basado en parámetros de Calidad de Servicio.

El programa de adiestramiento debe ser formulado como un sistema total, donde se derivan y toman en consideración las expectativas de los clientes para determinar las políticas de servicio en las cuales deben formarse los empleados, pero también deben detectarse las necesidades de entrenamiento del personal para fortalecer sus habilidades, destrezas en atención al público considerando sus competencias previas, y respetando sus aptitudes para la Calidad del Servicio.

Este último aspecto, será abordado en otra disertación como resultado final.

\section{Referencias bibliográficas}

Arias, Fidias (2006). El proyecto de investigación. Introducción a la metodología científica. Editorial Episteme. Caracas.

Atencio C., Edith y González P., Blanca (2006). Calidad de servicio en la editorial de la Universidad del Zulia (EDILUZ). Revista de Ciencias Sociales, abr. 2007, vol.13, no.1, p.172-186. ISSN 1315-9518.

Bounds, G. (1994). Beyond Total Quality Management, Mc Graw Hill. Bogotá.

Braidot, Néstor; Formento, Héctor y Nicolini, Jorge (2003). Desarrollo de una metodología de diagnóstico para empresas PYMES industriales $y$ de servicios: Enfoque basado en los sistemas de administración para la Calidad Total. Instituto de industrias argentinas. Buenos Aires.

Carlzon, Jan (1987). Moments of truch. Ediciones Harper and Row, Cambridge.

Crosby, Phill (1998). Competencia-PIenitudCalidad para el Siglo XXI. McGraw Hill. Bogotá.

González, O. (2001). La gerencia de la calidad en la Revista Pensamiento Estratégico. Ensayos. Ediciones El Gato Azul. Venezuela.

Deming, Edwar (1989). Calidad, productividad y competitividad, la salida de la crisis. Ediciones Diaz de Santos S.A. Madrid.

Hernández, Roberto; Fernández, Carlos y Baptista, Pilar (2006). Metodología de la Investigación. Cuarta edición. Mc Graw Hill, México.

Juran, Jaseph. y Gryna; Chief (1999). On Planning for Quality. Collier, USA: Mc Millan.

Kaplan, Robert; y Norton, David (2000). Cuadro de mando integral (the balanced scorecard). Ediciones Gestión, Barcelona.

Pestana, Lourdes y Garcia, Blanquita (2003). Gestión estratégica en la alcaldía del municipio Carirubana. Caso de estudio: La zona libre de inversión turística y comercial de la península de Paraguaná. Revista Venezolana de Ciencias Sociales, enero-junio.2003, vol.7, No. 1, p.10-31

Peters, Tom y Waterman, Robert (1987). En busca de la excelencia. Editorial Norma. Bogotá.

Rodríguez, Wilma (2000). Serie ISO 9000, Material de Apoyo. Congreso de Estudiantes de Ingeniería Industrial, Caracas- 2000 . 